\title{
Dynamic, state-dependent thresholds for the perception of single-element apparent motion: Bistability from local cooperativity
}

\author{
HOWARD S. HOCK, KRIS KOGAN, and JESSICA K. ESPINOZA \\ Florida Atlantic University, Boca Raton, Florida
}

\begin{abstract}
Previous studies have indicated that the formation of coherent patterns for multielement motion displays depends on global cooperative interactions among large ensembles of spatially distributed motion detectors. These interactions enhance certain motion directions and suppress others. It is reported here that perceiving one element moving between two nearby locations likewise is subject to cooperative influences (possibly facilitating and inhibiting interactions within a local ensemble of overlapping detectors). Thresholds depending on luminance contrast were measured for a generalized singleelement apparent-motion stimulus, and evidence for spontaneous switching and hysteresis effects indicated that motion perception near the $50 \%$ threshold was bistable. That is, for conditions in which motion and nonmotion were perceived half the time, the two percepts were distinct; when one was perceived, it clearly was discriminable from the other. These results indicated that (1) single-element apparent-motion thresholds depended on the immediately preceding state of the ensemble of motion detectors responding to the stimulus, and (2) the stimulus activation of individual motion detectors always might be influenced by recurrent, cooperative interactions resulting from the detectors' being embedded within interconnected ensembles.
\end{abstract}

Previous studies have indicated that the formation of coherent patterns for multielement motion displays depends on global cooperative interactions among ensembles of spatially distributed motion detectors that enhance certain motion directions and suppress others. Chang and Julesz (1983) and Williams and Sekuler (1984) have shown that all the dots in a random cinematogram appear to move coherently when a limited number of motion directions are stimulated consistently by a relatively small percentage of the moving elements, and Petersik (1990) has demonstrated that motion perceived in one part of a random cinematogram can increase the likelihood of motion being perceived in a neighboring part of the cinematogram. Nawrot and Sekuler (1990) and Hock and Balz (1994) have provided evidence that, depending on the distance, global spatial interactions can either facilitate or inhibit the perception of motion in a particular direction.

In this article, we report the results of experiments involving the perception of single-element apparent motion over small spatial displacements. When motion is not perceived, two stationary elements are perceived instead (the perception of nonmotion was sometimes, but not always, accompanied by the perception of flicker). The key finding is that the motion perceived for singleelement apparent motion is like the perception of global

We are grateful to Gregor Schöner, Kathleen Eastman, Martin Giese, and three anonymous reviewers for their careful reading of an earlier version of the manuscript and their valuable suggestions. Correspondence and requests for materials should be sent to H. Hock, Department of Psychology, Florida Atlantic University, Boca Raton, FL 33431 (e-mail: hockhs@fau.edu). motion in that both are subject to cooperative influences. As in the case of global motion, the cooperativity observed for single-element motion can be attributed to facilitating and inhibiting interactions among an ensemble of directionally selective motion detectors (Chang \& Julesz, 1983; Williams \& Sekuler, 1984). However, for singleelement motion, the cooperative interactions are likely to be limited to the local ensemble of overlapping, directionally selective motion detectors that is activated directly by the moving element. They cooperate with the effects of the stimulus on the motion detectors by enhancing relatively high levels of stimulus activation and/or suppressing relatively low levels of stimulus activation, thereby stabilizing competing motion and nonmotion percepts, reducing the likelihood of a spontaneous switch between them. As a result, the perception of both motion and nonmotion can be relatively stable near the $50 \%$ threshold. That is, the competing percepts can "resist" noisy fluctuations in sensory activation or changes in the value of stimulus parameters to values that favor the alternative percept.

It is implicit in classical psychophysical methods that perception near the $50 \%$ threshold is vague and uncertain. Much effort, therefore, has been concerned with developing analytic procedures to account for the guessing strategies (e.g., Blackwell, 1953) and changes in decision criteria (Green \& Swets, 1966) that appear under conditions of perceptual uncertainty. The latter, signal-detection approach, treats sensory noise as the source of perceptual uncertainty near the threshold. Signal and noise are additive effects on sensory activation, and the decision criterion (threshold) functions essentially as a passive, allor-none filter for deciding whether a signal is present 
(sensory activation passes through the filter) or not (sensory activation does not pass through the filter).

The research reported in this article was motivated by the observation of sudden, spontaneous switching (without any change in stimulus conditions) between the perception of motion and nonmotion for a generalized singleelement apparent-motion stimulus similar to a stimulus first described by Johansson (1950). Anstis, Giaschi, and Cogan (1985) and Finlay and Von Grünau (1987) have previously reported motion-to-nonmotion switches for single-element apparent motion and attributed the result to motion adaptation. Our observation of nonmotion-tomotion switches in addition to motion-to-nonmotion switches suggested that the two percepts could be bistable near the motion/nonmotion threshold; that is, the threshold is state dependent, or dynamic. ${ }^{1}$ The importance of this is that when the alternative percepts are sufficiently stable, the perception of motion versus nonmotion near threshold can be clear and distinct rather than vague and uncertain. From this point of view, the perceptual uncertainty near threshold that is an assumed characteristic of classical psychophysical methods would be expected only under conditions for which the alternative percepts were so unstable that neither would be realized in perceptual experience long enough for a distinct percept to be established (Schöner \& Hock, 1995).

The effects of sensory noise remain an essential aspect of dynamical thresholds, but if the only effect of the noise were to randomly add to or subtract from the sensory activation due to the signal, the situation would be no different from that described for signal-detection methods. The dynamical approach is distinctive, however, because the effects of signal and noise are not additive. That is, perceptual switching may be caused by the presence of sufficiently high levels of sensory noise, but cooperative interactions arising from the visual system's response to the signal could result in the formation of percepts that "resist" the effects of more moderate noise levels. Such percepts then would have the quality of being temporally stable. As a result, one or the other would be realized in perceptual experience long enough to be perceptually distinct, even near threshold.

Three experiments are reported in this study. The purpose of the first was to identify the stimulus parameter that controls the motion/nonmotion threshold for the generalized single-element apparent-motion stimulus. This parameter, which involves luminance contrast, was then manipulated in the second and third experiments in order to provide evidence for the dynamic, state-dependent quality of perception near the $50 \%$ threshold. Experiment 2 measured the temporal stability of competing motion and nonmotion percepts. Experiment 3 measured hysteresis effects.

\section{EXPERIMENT 1}

In most studies of single-element apparent motion, one element is presented at a time, first in one spatial lo- cation, then in another. Because motion is perceived over a wide range of spatial and temporal parameters, measurement usually has been limited to judgments of motion quality or motion strength (e.g., Attneave, 1971; Kolers, 1972). Illustrative is what may be the first demonstration of state dependence in motion perception (Sekuler, 1996; Wertheimer, 1912): When an apparent motion stimulus with an interstimulus interval that was judged to yield "good" motion perception preceded one with an interstimulus interval that was judged to yield "poor" motion perception, Wertheimer's observers tended to see "good" motion for the otherwise "poor" motion stimulus (and vice versa when a "poor" apparent motion stimulus preceded a "good" apparent motion stimulus).

A potential difficulty, however, lies in consistently defining such inherently subjective qualities as "good" and "poor" for different observers, different experiments, and different laboratories. This is made evident in another experiment in which observers comparing real and apparent motion sometimes reported that the apparent rather than the real motion was the best movement of all (Sekuler, 1996; Wertheimer, 1912). As an alternative to judgments of motion "goodness" or motion "strength," we have measured apparent-motion thresholds with a generalized version of the single-element apparent-motion stimulus for which subjects discriminate between the perception of motion and nonmotion.

For the generalized single-element apparent-motion stimulus, two elements can be seen simultaneously, each with a luminance that is different from the background luminance. Motion between the two elements (small squares) can be perceived when their luminance values are exchanged on successive frames. A stimulus very similar to this was described by Johansson (1950). Instead of discrete exchanges in luminance on successive frames, he sinusoidally varied the luminance of two nearby circular areas, introducing a phase difference of $180^{\circ}$ between the luminance variations at each location. Johansson's observers reported seeing a light with constant brightness moving through the space between and behind the stimulated circular areas, which were perceived as semitransparent windows. Although the stimuli presented in the current study sometimes resulted in a similar sort of aperture motion, the subjects were instructed to respond positively only when they perceived object motion, that is, when one of the small squares was perceived as moving through the space between them. In additional experiments, we have found that the perception of motion behind perceptually semitransparent apertures is eliminated when the squares are sufficiently small, and furthermore, that similar results to those reported in this article are obtained when the possibility of perceiving aperture motion is eliminated. The comparison of perceived aperture motion with perceived object motion will be the subject of a future article.

It should be noted that standard apparent motion (only one of the two elements is visible at a time) is a special case of the more general single-element apparent-motion 
paradigm studied in this article. That is, in the general paradigm, the luminance values of the two simultaneously presented elements are different, the luminance values are exchanged on successive frames, and both luminance values are different from the background luminance (it is called single-element motion because only one element is perceived as moving). In the special case of standard apparent motion, the lower luminance value always corresponds to the background luminance. Another special case of the more general single-element apparent-motion paradigm is the reversed luminance polarity stimuli studied by Anstis and Mather (1985); for their stimuli, the background luminance was intermediate to that of the two simultaneously visible visual elements (one was lighter than background, the other, darker than background). They found that it was the element most dissimilar in luminance from that of the background that was perceived as moving, and furthermore, that nonmotion was perceived when both elements were equally dissimilar from the background. With the exception of a subset of stimuli in Experiment 2, both of the small squares presented in the current study were lighter than the background. Consistent with Anstis and Mather's results, the lighter of the two squares was perceived as moving through the space between them. ${ }^{2}$

What is critical for the current study is that when differences in the luminances of the squares are relatively small, nonmotion rather than motion is perceived. Since the motion and nonmotion percepts are easy to discriminate, reliable psychophysical judgments for the perception of single-element apparent motion could be obtained over a wide range of luminance values.

\section{Method}

Stimuli and Design. Two horizontally separated (42' apart), $4.2^{\prime} \times 4.2^{\prime}$ squares with different luminance values were presented simultaneously in the center of a gray rectangular background $\left(6.7^{\circ} \times 3.3^{\circ}\right)$ that was centered in the darkened $\left(1 \mathrm{~cd} / \mathrm{m}^{2}\right)$ screen of a Macintosh II 13-in. RGB monitor (a head restraint maintained the viewing distance at $30 \mathrm{~cm}$ ). The luminance of the rectangular background $\left(L_{b}\right)$ was discriminated easily from that of the surrounding computer screen. $L_{\mathrm{b}}$ was $2.2,3.3$, or $4.5 \mathrm{~cd} / \mathrm{m}^{2}$ during three separate blocks of 108 randomly ordered trials (the order of these blocks was counterbalanced during each of the six testing sessions). At each location of the apparent-motion stimulus, $L_{\mathrm{m}}$, the approximate average luminance, ${ }^{3}$ was $6.6,7.7$, or $8.9 \mathrm{~cd} / \mathrm{m}^{2}$, and $L_{1}-L_{2}$, the luminance difference, was $0.4,0.8,1.1,1.5,1.9,2.3,2.7,3.1$, or $3.4 \mathrm{~cd} / \mathrm{m}^{2}$. The order of the 27 stimuli generated by the orthogonal combination of $L_{\mathrm{m}}$ and $\left(L_{1}-L_{2}\right)$ was randomized in subblocks of 27 trials. The two luminance values of each square were exchanged on alternating 195-msec frames.

Procedure. Subjects were instructed to fixate midway between the two squares (there was no visible fixation point) and, after each 20 -frame trial, to press one of two computer keys (without speed stress) to indicate whether or not motion had been perceived anytime during the trial. They were instructed to respond positively if a square appeared to be moving through the space between the actual locations of the squares, and to respond negatively when the squares looked like stationary apertures behind which motion (of an ill-defined shape) was perceived. Although the instructions did not distinguish between motion of the lighter versus the darker square (or both simultaneously), postexperimental subjective reports indicated that the perceived motion was always of the lighter square.
}

Subjects. Two of the authors and a naive undergraduate student (a 22-year-old female) with no previous experience in psychophysical experiments served as subjects. All had normal or-corrected-tonormal vision.

\section{Results}

Nine psychometric functions were obtained for each subject (Figure 1) by varying the squares' luminance difference $\left(L_{1}-L_{2}\right)$, average luminance $\left(L_{\mathrm{m}}\right)$, and background luminance $\left(L_{\mathrm{b}}\right)$. These functions approximately covered the entire space of luminance values studied in this experiment. Motion perception increased with increases in the luminance difference $\left(L_{1}-L_{2}\right)$, decreases in the average luminance $\left(L_{\mathrm{m}}\right)$, and increases in the background luminance $\left(L_{\mathrm{b}}\right)$. However, whether or not motion was perceived was predicted most directly by a single variable, $\left(L_{1}-L_{2}\right) \div\left(L_{\mathrm{m}}-L_{\mathrm{b}}\right)$, the background-relative luminance contrast (BRLC). As can be seen in the far right graphs of Figure 1, when the proportion of trials for which motion was perceived was graphed on the basis of the BRLC value for each stimulus, the nine functions for each subject collapsed into a single psychometric function with a steep slope. Probit-determined 50\% thresholds (the BRLC was approximately equal to 0.5 ) are presented with each graph.

\section{Discussion}

The results of this experiment indicated that whether motion or nonmotion was perceived for the generalized single-element apparent-motion stimulus depended on the BRLC, which is the ratio of the time-varying $\left(L_{1}-L_{2}\right)$ and time-invariant $\left(L_{\mathrm{m}}-L_{\mathrm{b}}\right)$ luminance characteristics of the apparent-motion stimulus. In effect, the extent to which time-varying components provide evidence for spatial changes in the visual world (motion) is tempered by the extent to which time-invariant components provide evidence that nothing has changed. The BRLC served as the parameter controlling the stability of the motion and nonmotion percepts in Experiments 2 and 3 .

\section{EXPERIMENT 2}

The purpose of this experiment was to determine whether the motion and nonmotion percepts formed for the generalized single-element apparent-motion stimuli were temporally stable near the $50 \%$ threshold. Temporal stability here means that the percept resists spontaneous change to the competing percept despite the presence of noisy sensory fluctuations that would encourage such a change. Thus, a threshold is dynamic when what is perceived at a given moment depends on the preceding perceptual state; that is, the likelihood of perceiving motion during a pair of frames is increased when motion is perceived during the preceding pair of frames.

\section{Method}

As in the first experiment, apparent-motion stimuli were formed by simultaneously presenting two $4.2^{\prime} \times 4.2^{\prime}$ squares with different luminance values, and exchanging their luminance values on suc- 


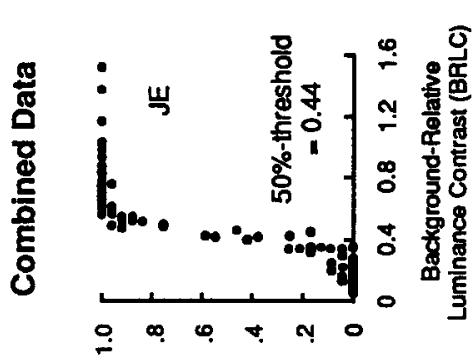

voliow penjeosed volpuodasd

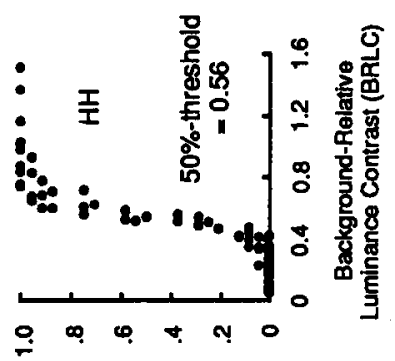

uopow penjesed uophodold

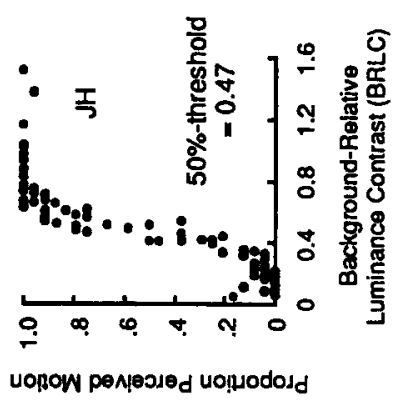

焉

昰

5

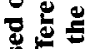

产

政

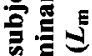

娄贯

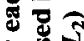

s

可

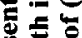

象

용

政

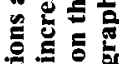

政

政

2

5 to

政.

喝

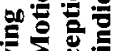

绕

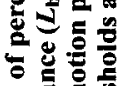

要要

政

至要。

콜

焉焉

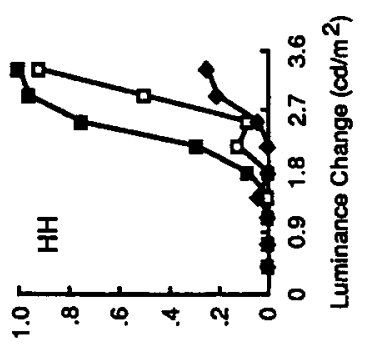

uonow pespesed uoluodosd

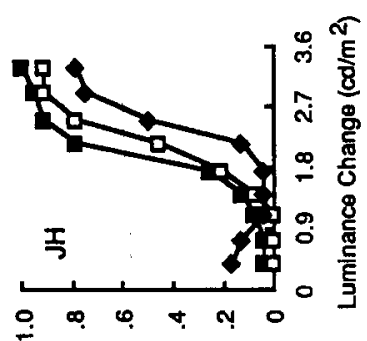

uolnow peniesed uoluodold 
cessive $195-\mathrm{msec}$ frames. The stimuli varied with respect to $L_{1}-L_{2}\left(0.7,1.4,2.1,2.7,3.4\right.$, or $\left.4.1 \mathrm{~cd} / \mathrm{m}^{2}\right)$ and $L_{\mathrm{m}}$ (approximately $4.1,6.2,8.2$, or $\left.10.3 \mathrm{~cd} / \mathrm{m}^{2}\right) . L_{\mathrm{b}}$ was $4.1 \mathrm{~cd} / \mathrm{m}^{2}$ for every trial. One of 18 apparent-motion stimuli was presented during each trial. For 16 of these stimuli, both squares were visible simultaneously, including 4 for which $L_{\mathrm{m}}$ approximately equaled $L_{\mathrm{b}}$, so that one square was lighter than the background and the other square was darker than the background (as in Anstis \& Mather, 1985). The remaining two stimuli were standard apparent-motion stimuli; only one square was visible at a time $\left(L_{2}=L_{\mathrm{b}}\right)$.

After each 20-frame trial, subjects first pressed one of two computer keys (without time stress) to indicate whether motion or nonmotion was perceived at the start of the trial, and then pressed one of two other keys to indicate whether there had been a change to the other percept anytime during the trial. There were four daily sessions, each with two blocks of 72 trials (randomly ordered within subblocks of 18 trials).

The subjects were one of the present authors and two naive undergraduate students (one male and one female, both 24 years old).

\section{Results}

Motion versus nonmotion at the start of each trial. When the BRLC was relatively small, nonmotion usually was perceived at the start of the trial; when it was relatively large, motion usually was perceived at the start of the trial. The graphs on the left side of Figure 2 indicate the steep psychometric functions and thresholds that previously were observed in Experiment 1 . The dependence of motion perception on the BRLC therefore was similar, regardless of whether subjects' responses specified when the percept occurred (at the start of the trial in Experiment 2) or whether they did not specify when it occurred (anytime during the trial in Experiment 1).

Included in the experiment were standard apparentmotion stimuli ( $L_{2}=L_{\mathrm{b}}$, so only one square was visible at a time), for which BRLC $=2.0$, regardless of the values of $L_{1}, L_{2}$, and $L_{\mathrm{b}}$. Since this value substantially exceeds threshold, motion always was perceived, as it consistently is perceived for standard apparent-motion stimuli over a wide range of displacements and temporal frequencies (Kolers, 1972). The latter assumes, of course, that the elements are sufficiently different in luminance from the background to be detectable.

Also included in the experiment were apparentmotion stimuli for which $L_{\mathrm{m}}$ approximately equaled $L_{\mathrm{b}}$ (at $4.1 \mathrm{~cd} / \mathrm{m}^{2}$ ). For these stimuli, which are like those studied by Anstis and Mather (1985), the luminance polarity reversed on successive frames (from lighter than background to darker than background, and so on), and motion almost always was perceived. Anstis and Mather found, for stimuli like these, that it is the element most dissimilar in luminance from the background that is perceived as moving. Since motion generally was perceived in the current study for the lighter-than-background square, it is likely, in most cases, that $L_{\mathrm{m}}$ was somewhat larger than $L_{\mathrm{b}}$ (recall from Footnote 1 that the average luminance values could not be exactly matched). The fact that motion occasionally was not perceived was consistent with Anstis and Mather's further report that motion perception was eliminated when the two visual elements were equally different from the background luminance
$\left(L_{\mathrm{m}}=L_{\mathrm{b}}\right)$. The absence of motion perception may have been due to mutual inhibition among motion detectors that respond independently to opposing luminance polarities (Raymond \& Braddick, 1996). Because $L_{\mathrm{m}}$ and $L_{\mathrm{b}}$ were not matched exactly, the data for these stimuli were placed between the 2.0 and $\infty$ values on the BRLC axis of Figure 2 (BRLC $=\infty$ when $L_{\mathrm{m}}=L_{\mathrm{b}}$, but would equal 2.0 if motion perception was based on independent motion detectors for lighter than background and darker than background stimuli).

Switching and state dependence. Although the initial percept often remained unchanged for an entire trial, spontaneous switching from motion to nonmotion, or vice versa, often was perceived. The scattergrams for perceptual switching are presented in Figure 2. Each point in the three scattergrams in the middle of Figure 2 (motion-to-nonmotion switches) represents the proportion of trials for which the initial percept was motion and the proportion of these initial-motion trials for which there subsequently was at least one spontaneous switch to the perception of nonmotion. Each point in the three scattergrams on the right side of Figure 2 (nonmotion-tomotion switches) represents the proportion of trials for which the initial percept was nonmotion and the proportion of these initial-nonmotion trials for which there subsequently was at least one spontaneous switch to the perception of motion. The number of switches per trial and the proportion of each trial's duration with and without the perception of motion were not assessed.

Despite there being 18 possible points in each scattergram (corresponding to the 18 different apparent-motion stimuli generated by combinations of $L_{1}-L_{2}$ and $L_{\mathrm{m}}$ ), the motion-to-nonmotion scattergrams have fewer points, because there were many stimuli for which motion was never the initial percept (e.g., initial motion usually was not perceived on trials with very small BRLC values). Similarly, the nonmotion-to-motion scattergrams have fewer than 18 points, because there were many trials for which nonmotion was never the initial percept (e.g., initial nonmotion usually was not perceived on trials with very large BRLC values). In addition, some points were hidden because they occupied identical locations on the scattergrams.

As can be seen for both motion-to-nonmotion and nonmotion-to-motion switches in Figure 2, the greater a percept's likelihood at the start of a trial, the less the likelihood of a spontaneous switch to the competing percept later during the trial. Percepts occurring at the start of a trial that are favored by the BRLC value (e.g., motion for high BRLCs) are highly stable, and therefore relatively unlikely to switch. Conversely, percepts occurring at the start of a trial, even though they are not favored by the BRLC value (e.g., motion for low BRLCs), are highly unstable, and therefore relatively likely to switch. Thus, the likelihood of a switch is state dependent; it depends on whether the current percept state is established strongly or weakly by the activating stimulus conditions.

Highlighted in the scattergrams are points for which the measured proportions of switches from the initial 

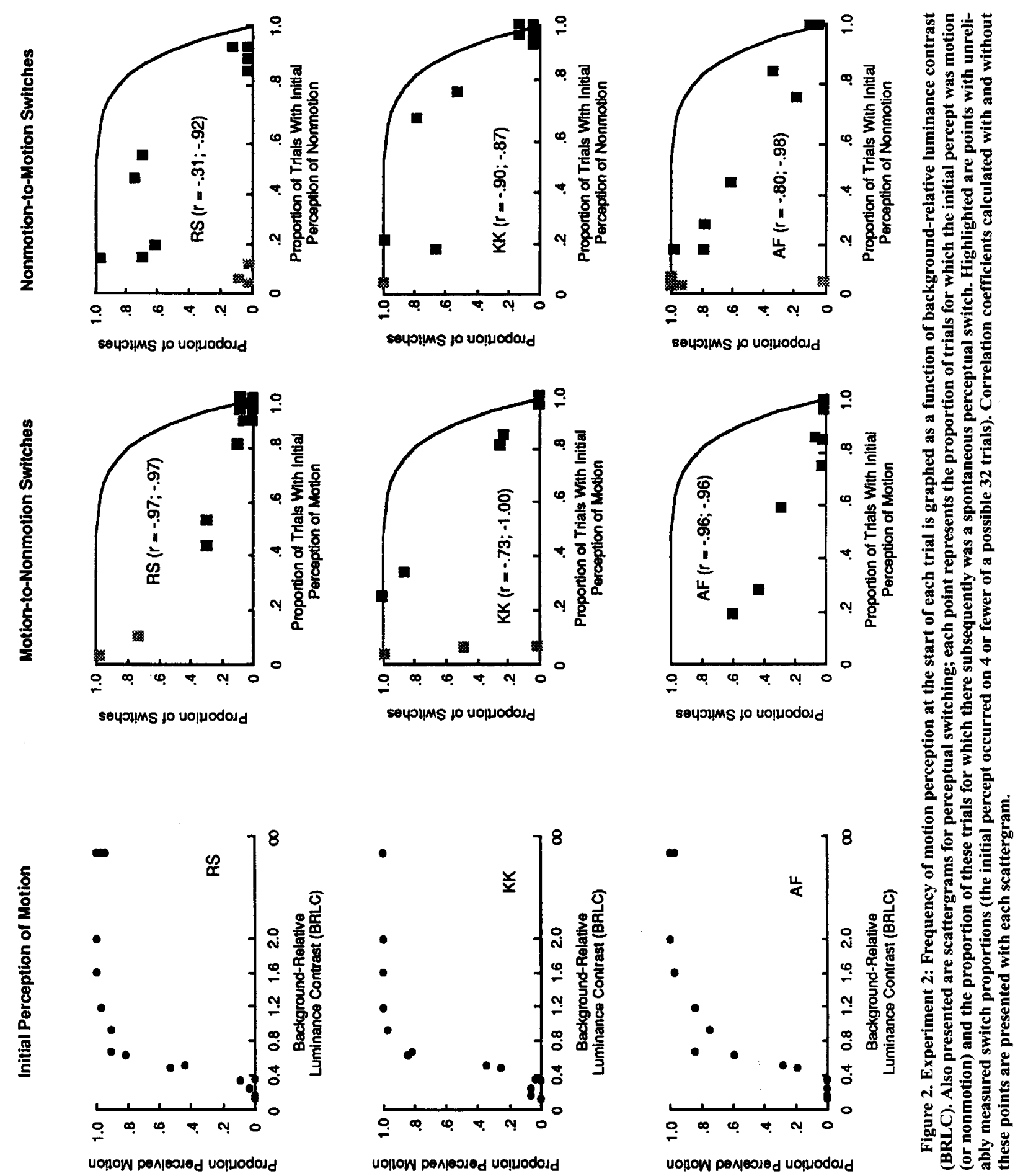

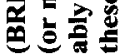


percept were unreliable; the initial percept for these points occurred on 4 or fewer of 32 possible trials, so estimates of switching proportions were based on very few trials. Correlation coefficients calculated with and without these points were consistently negative (they are presented with each scattergram).

State dependence near the $50 \%$ threshold. It can be seen in the middle graphs of Figure 2 that when motion was perceived at the start of approximately $50 \%$ of the trials, the probability of a switch was intermediate to that observed for more extreme initial probabilities. The same was true when nonmotion was perceived at the start of approximately $50 \%$ of the trials (the graphs on the right side of Figure 2). The critical question, therefore, is whether these intermediate switching rates reflect the state dependence of the percept (zero switching probabilities are not necessary for a percept to be state dependent) or whether the occurrence of spontaneous switching involves nothing more than the independent opportunity for motion or nonmotion to be seen on every pair of frames in the 20 -frame trial. In the latter case, perception for any pair of frames would not depend on whether the perceptual state during the preceding frames was motion or nonmotion.

On the assumption of frame-to-frame independence, the probability of a switch from the initially formed (during the first pair of frames) to the competing percept (during one of the nine succeeding pairs of frames) was calculated on the basis of the probability, $p_{1}$, of the initial percept: $p_{\text {switch }}=\left(1-p_{1}^{9}\right)$. The resultant independentof-previous-state function is superimposed on each scattergram in Figure 2 ; at the $50 \%$ threshold $\left(p_{1}=.5\right)$, $p_{\text {switch }}=.998$. As can be seen in Figure 2 , in the vicinity of the $50 \%$ threshold, the probability of a switch from motion to nonmotion, or vice versa, was much less than what would be expected on the basis of the assumption of frame-to-frame independence. Thus, both the motion and nonmotion percepts near the $50 \%$ threshold were relatively stable (state dependent); for example, the perception of motion (nonmotion) during a pair of frames was made more likely when motion (nonmotion) was perceived during the preceding pair of frames.

\section{EXPERIMENT 3}

The dynamic (state-dependent) character of perception near the $50 \%$ threshold was examined further by gradually increasing or gradually decreasing the BRLC on successive frames within a trial and comparing the results to trials for which the BRLC was constant for the entire trial. Hysteresis effects (free of response artifacts) would provide evidence that either motion or nonmotion could be perceived with high frequency near the threshold, depending on whether the BRLC was gradually increased or gradually decreased.

\section{Method}

A modified version of the classical method of limits (Hock, Kelso, \& Schöner, 1993) was used to eliminate "habituation" or response bias effects that are artifacts in the classical method (e.g.,
Corso, 1967). Instead of responding following each change in parameter value, subjects in the modified method do not respond until the end of each ascending or descending trial. They indicate, without speed stress, whether there had been a perceptual switch from motion to nonmotion, or vice versa, anytime during the trial. When these switches occurred was found by randomly varying the endpoint BRLC of each trial, which determined how "deeply" each ascending or descending trial had penetrated the transitional (bistable) region of the BRLC. For example, a descending trial with an end-point BRLC value of 0.6 was composed of frame pairs with the following sequence of BRLC values: $0.9 \rightarrow 0.8 \rightarrow 0.7 \rightarrow 0.6$. Deeper penetration into the transition zone was achieved for descending trials with end-point BRLC values of 0.4 , which were composed of frame pairs with the following sequence of BRLC values: $0.9 \rightarrow 0.8 \rightarrow 0.7 \rightarrow 0.6 \rightarrow 0.5 \rightarrow 0.4$. More perceptual switches were anticipated for trials probing deeper into the transition zone.

With $L_{\mathrm{b}}=3.3 \mathrm{~cd} / \mathrm{m}^{2}$, nine BRLC values, ranging from 0.1 to 0.9 , were created by varying $L_{1}-L_{2}$ while holding $L_{\mathrm{m}}$ approximately constant at $7.0 \mathrm{~cd} / \mathrm{m}^{2}$. The $L_{1} / L_{2}$ pairs, $7.2 / 6.8,7.3 / 6.6,7.5 / 6.4$, $7.7 / 6.2,7.9 / 6.1,8.1 / 5.9,8.3 / 5.7,8.5 / 5.5$, and $8.7 / 5.4 \mathrm{~cd} / \mathrm{m}^{2}$ were changed by one step every two frames (the size of each square was increased to $8.4^{\prime} \times 8.4^{\prime}$ in this experiment). Randomly intermixed were trials for which the BRLC remained constant during an entire 10-frame trial. The frame duration was $195 \mathrm{msec}$ in all conditions. There were three testing sessions (three blocks of 100 trials per session). The subjects were one of the authors and a naive undergraduate student (a 21-year-old female).

\section{Results}

Hysteresis in motion perception has previously been observed for competition between the perception of coherent and incoherent motion (Williams, Phillips, \& Sekuler, 1986) and competition between the perception of horizontal and vertical motion (Hock et al. 1993). It was observed in the present study for competition between the perception of single-element apparent motion and the perception of nonmotion.

In Figure 3, the proportion of descending trials (BRLC values were reduced during every other frame) for which motion was perceived without any switches to the perception of nonmotion is graphed with respect to the axis on the left of the graphs. Also graphed with respect to the axis on the left is the proportion of constant trials (BRLC values remained constant within a trial) for which motion was perceived without any switches to the perception of nonmotion during the trial. The proportion of ascending trials (BRLC values were increased during every other frame) for which nonmotion was perceived without any switches to the perception of motion is graphed with respect to the inverted axis on the right of the graphs. Also graphed with respect to the inverted axis on the right is the proportion of constant-BRLC trials for which nonmotion was perceived without any switches to the perception of motion during the trial. Since the number of trials with motion-to-nonmotion switches was not constrained to be the same as the number of trials with nonmotion-to-motion switches, the proportion of motion-without-switching trials (axis on the left) was not the reciprocal of the proportion of nonmotionwithout-switching trials (axis on the right).

In the absence of hysteresis, the results for the descending and ascending trials would have overlapped. That is, initially perceived motion would have switched to the 


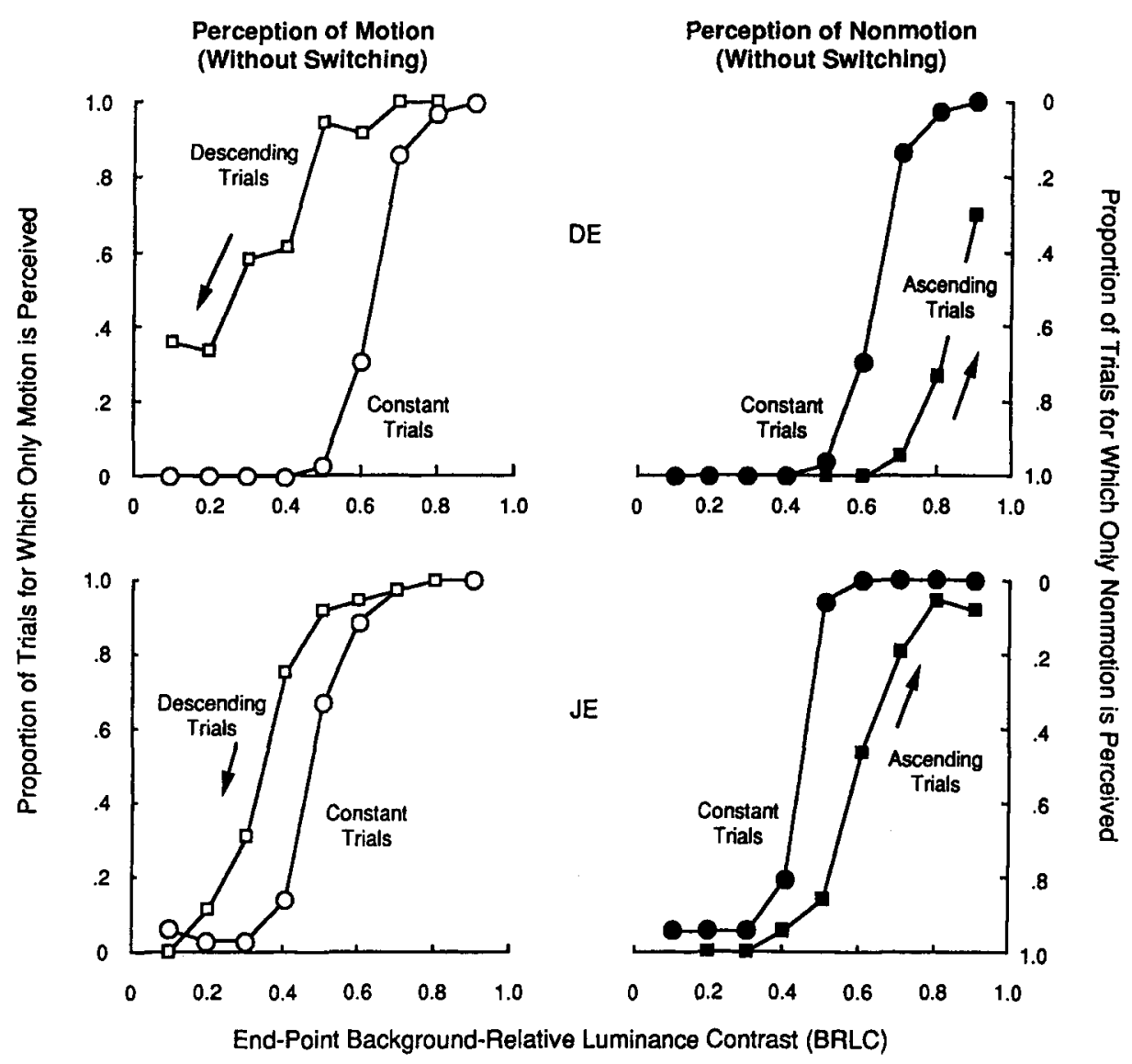

Figure 3. Experiment 3: Hysteresis effects were assessed by gradually increasing or gradually decreasing the background-relative luminance contrast (BRLC) and varying the end-point BRLC value for each trial. The proportion of descending-BRLC and constant-BRLC trials for which motion was perceived without switching is indicated by the axis on the left side of the graphs. The proportion of ascending-BRLC and constant-BRLC trials for which nonmotion was perceived without switching is indicated by the inverted axis on the right side of the graphs.

perception of nonmotion on $50 \%$ of the descending trials for the same end-point BRLC value as initially perceived nonmotion switched to the perception of motion on $50 \%$ of the ascending trials. However, the results provided evidence for the presence of hysteresis; whether motion or nonmotion was perceived for a particular endpoint BRLC value (in the transition region) depended on the direction in which the BRLC was changed, and thus, the preceding perceptual state. As can be seen by comparing the graphs on the left and right side of Figure 3, motion was perceived for the entirety of $80 \%-95 \%$ of the descending trials starting with high BRLC values and ending with values in the vicinity of the $50 \%$ threshold (BRLC of approximately 0.5 ), whereas nonmotion was perceived for the entirety of $90 \%-100 \%$ of the ascending trials, starting with small BRLC values and ending with BRLC values in the vicinity of the $50 \%$ threshold. ${ }^{4}$

In the constant condition (the BRLC remained constant for the entire trial), we determined the proportion of trials for which motion was perceived (without switching to nonmotion) and the proportion of trials for which nonmotion was perceived without switching to motion.
Since spontaneous switches were possible in the constant-BRLC condition (they were sometimes observed for J.E., but not for D.E.), the two constant-BRLC functions in Figure 3 were not constrained to be the same. When descending trials were compared with constant trials (graphs on the left side of Figure 3), there were more trials for which motion was perceived (without switching to nonmotion) for the descending trials. This indicated that the likelihood of perceiving motion for a particular BRLC was enhanced when the motion percept was established beforehand by a higher BRLC value than when it was established beforehand by the same BRLC value. This provided further evidence for state dependence of the motion percept; the likelihood of its continued perception during a trial depended on how strongly the motion state was established by the activating stimulus conditions during the immediately preceding pair of frames.

When ascending trials were compared with constant trials (graphs on the right side of Figure 3), there were more trials for which nonmotion was perceived (without switching to motion) for the ascending trials. This indicated that the likelihood of perceiving nonmotion for a particular 
BRLC was enhanced when the nonmotion percept was established beforehand by a lower BRLC value than when it was established beforehand by the same BRLC value. This provided further evidence for state dependence of the nonmotion percept; the likelihood of its continued perception during a trial depended on how strongly the nonmotion state was established by the activating stimulus conditions during the immediately preceding pair of frames.

\section{Additional Results}

An additional experiment was conducted in order to confirm that the hysteresis effect observed in this experiment was associated with each trial's end-point BRLC value rather than its duration (e.g., ascending trials with an end-point BRLC value of 0.3 were briefer than ascending trials with an end-point BRLC value of 0.9 ). In this experiment, the total duration of every trial was the same, nine pairs of $195-\mathrm{msec}$ frames. For example, in the primary version of Experiment 3, the longest duration ascending trial started with a BRLC value of 0.1 and ended after nine pairs of frames with a BRLC value of $0.9(0.1 \rightarrow 0.2 \rightarrow 0.3 \rightarrow 0.4 \rightarrow 0.5 \rightarrow 0.6 \rightarrow 0.7 \rightarrow 0.8$ $\rightarrow 0.9)$. To maintain a constant trial duration of nine pairs of 195-msec frames, ascending trials with small end-point values had a compensating number of frames for which the initial BRLC value (0.1) was repeated, and descending trials with large end-point values had a compensating number of frames for which the initial BRLC value (0.9) was repeated. For example, the starting value of ascending trials with an end-point value of 0.3 was presented for seven pairs of frames, so that the trial again was composed of nine pairs of 195-msec frames $(0.1 \rightarrow$ $0.1 \rightarrow 0.1 \rightarrow 0.1 \rightarrow 0.1 \rightarrow 0.1 \rightarrow 0.1 \rightarrow 0.2 \rightarrow 0.3)$. Randomly intermixed with the descending and ascending trials were trials for which the BRLC remained constant during an entire trial (nine pairs of 195-msec frames).
The results were in close agreement with those obtained in the primary experiment (Figure 4).

\section{Discussion}

Despite changes in the BRLC to values favoring the competing percept, the stability of the initially formed motion and nonmotion percepts reflected the dynamic character of perception near the $50 \%$ threshold; that is, in addition to the effect of the stimulus on the motion detectors, what is perceived is influenced by the immediately preceding perceptual state of the motion-detecting system. If motion has just been perceived, it is likely to continue being perceived. The additional results, obtained with trial duration controlled, showed that the persistence of the percept established at the start of each trial depended on how far the ascending or descending BRLC value penetrated into the range of values favoring the competing percept and not on the duration of the trial.

\section{GENERAL DISCUSSION}

The results of Experiments 2 and 3 provided evidence for the state dependence of the motion and nonmotion percepts formed for the generalized single-element apparent-motion stimulus. Since the "stabilization" of these percepts near threshold occurred for small, closely spaced stimuli (small squares $42^{\prime}$ apart), local cooperative effects that enhance and/or suppress stimulusdependent detector activation were implicated. Although the reported experiments are not definitive regarding the source of the cooperativity, studies involving global cooperativity (Chang \& Julesz, 1983; Hock \& Balz, 1994; Nawrot \& Sekuler, 1990; Petersik, 1990; Williams et al. 1986; Williams \& Sekuler, 1984) point to the role of long-range (Gilbert, 1985) facilitating and inhibiting interactions among directionally selective motion detec-
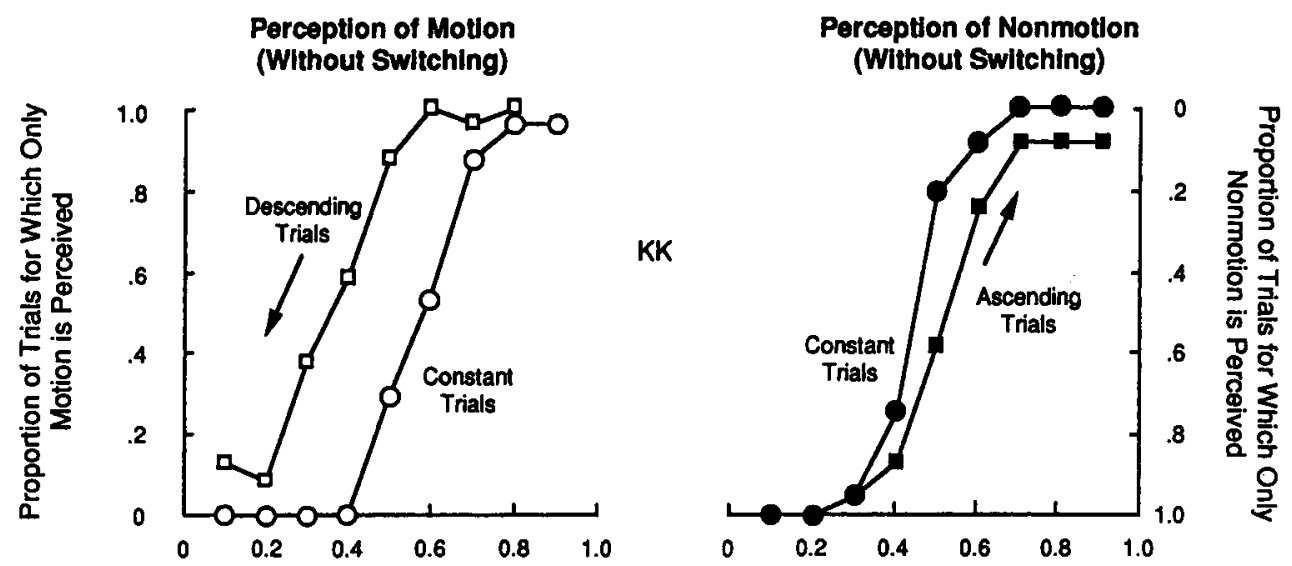

End-Point Background-Relative Luminance Contrast (BRLC)

Figure 4. Experiment 3 (additional results): Hysteresis effects obtained when the total duration of each ascending and descending trial was the same, regardless of the end-point background-relative luminance contrast (BRLC) value. The proportion of descending-BRLC and constant-BRLC trials for which motion was perceived without switching is indicated by the axis on the left side of the graphs. The proportion of ascending-BRLC and constant-BRLC trials for which nonmotion was perceived without switching is indicated by the inverted axis on the right side of the graphs. 
tors. Similar, but shorter range, interactions among the overlapping, spatially local ensemble of detectors directly activated by the single-element apparent-motion stimulus could likewise account for the cooperativity observed in the current study. When a motion detector is strongly activated by the stimulus (high BRLC), the activation can be further enhanced by facilitating interactions with other strongly activated motion detectors with similar directional selectivity. However, when the stimulus activation of a motion detector is weak (low BRLC), facilitating interactions are weak and, therefore, can be overwhelmed by inhibiting interactions with other weakly activated motion detectors (assuming that the ensemble of mutually inhibiting detectors is larger than the ensemble of mutually facilitating detectors; Hock, Balz, \& Eastman, 1996). Motion-detector activation would then be suppressed, and the nonmotion percept realized. ${ }^{5}$

If the thresholds measured in this study were nondynamic, the likelihood of motion perception would be expected to vary smoothly with activation level (see the hy- pothetical activation functions in Figure 5). Thereupon, when activation was slightly above the nondynamic $50 \%$ threshold, even a small stochastic fluctuation would have been sufficient to reduce it below threshold, causing a perceptual switch from motion to nonmotion. Switches in both directions would have occurred so often that both percepts would have been highly unstable, and perception would have been vague and uncertain.

The "forcing apart" of activation levels by cooperative interactions, which provides the essential difference in the hypothetical activation functions of dynamic as opposed to classical, nondynamic thresholds, can be represented by a bifurcation in the dynamic activation function (Figure 5). The upper branch of the dynamic activation function reflects the cooperative enhancement of relatively high, stimulus-dependent activation levels, perhaps by recurrent facilitating interactions. The lower branch reflects the cooperative suppression of relatively low, stimulus-dependent activation levels, perhaps by recurrent inhibiting interactions. Because of these cooper-
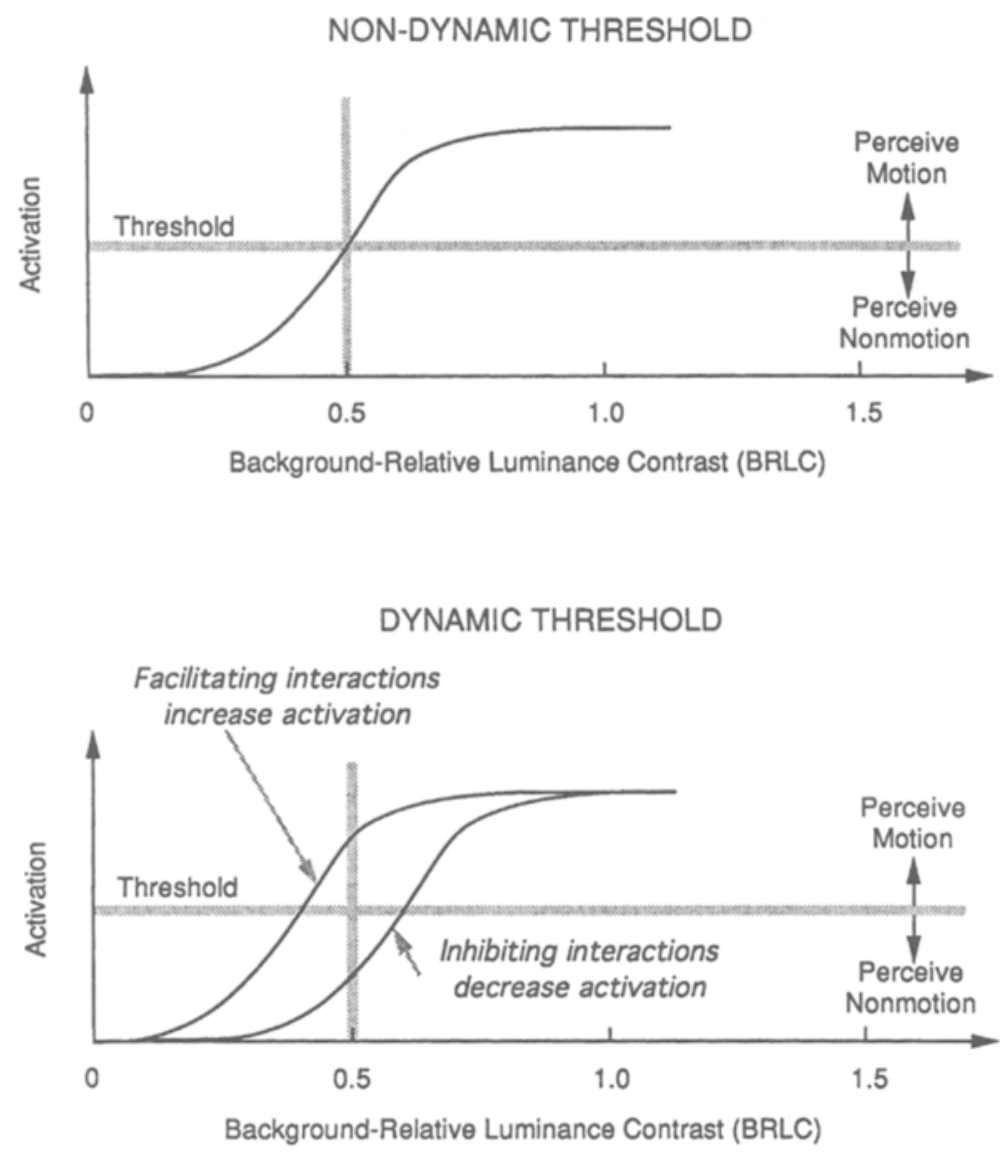

Figure 5. Hypothetical functions relating the activation of motion detectors to the perception of motion for nondynamic and dynamic thresholds. For nondynamic thresholds, the likelihood of motion perception varies smoothly with activation level. The hypothetical activation function for dynamic thresholds is bifurcated, the upper branch reflecting the cooperative enhancement of high, stimulus-dependent activation levels by recurrent facilitating interactions, and the lower branch reflecting the cooperative suppression of low, stimulus-dependent activation levels by recurrent inhibiting interactions. 
ative effects, when motion and nonmotion each were perceived approximately half the time (near the 50\% threshold), activation was either substantially above or substantially below threshold, so, in contrast to nondynamic thresholds, only relatively large stochastic fluctuations produced spontaneous perceptual switches. Since large fluctuations occur relatively infrequently, the motion and nonmotion percepts were relatively stable, and therefore realized long enough in perceptual experience to be perceptually distinct.

How long a pattern persists in perceptual experience before it loses its stability depends on whether it is formed under conditions favoring or disfavoring it. When the BRLC is substantially above threshold, strong stimulus activation of motion detectors is likely to be cooperatively enhanced, placing the local ensemble of motion detectors on the upper branch of the bifurcated activation function (see Figure 5). Motion perception, which then is likely, would be resistant to spontaneous change since a stochastic fluctuation large enough to reduce activation below threshold will occur infrequently (see points falling in the lower right corner of the "motion-tononmotion" scattergrams in Figure 2). When the BRLC is substantially below threshold, stimulus activation of motion detectors is relatively weak, and it is unlikely that the local ensemble of motion detectors will lie on the cooperatively enhanced upper branch. However, on those relatively infrequent occasions when it does lie on the upper branch and motion is perceived, the motion percept will be unstable because a small stochastic fluctuation will be sufficient to reduce activation below threshold (see Figure 5 as well as points falling closer to the upper left corner of the "motion-to-nonmotion" scattergrams in Figure 2).

The dynamic activation function also accounts for the observed hysteresis effects (Figures 3 and 4). For example, when a trial begins with a large value of BRLC, motion-detector activation (already relatively high) is likely to be enhanced further by recurrent facilitating interactions so that, when the BRLC is decreased gradually, activation is likely to be determined by the upper branch of the bifurcated activation function. As a result, motion will be perceived at the start of each descending trial and continue to be perceived even as the BRLC is decreased to values that would otherwise favor the perception of nonmotion. Conversely, when a trial begins with a small value of BRLC, motion-detector activation (already relatively low) is likely to be reduced further by recurrent inhibiting interactions. Therefore, when the BRLC is increased gradually, activation is likely to be determined by the lower branch of the bifurcated activation function, so nonmotion will be perceived at the start of each descending trial and continue to be perceived even as the BRLC is increased to values that would otherwise favor the perception of motion.

In conclusion, the evidence for state dependence obtained in Experiments 2 and 3 suggests that local cooperativity influences the perception of single-element apparent-motion. This implies that the effects of a stimulus on individual motion detectors cannot be assessed outside the context of the interconnected, interactive ensemble of detectors in which they are embedded. That is, the effects of a stimulus on motion-detector activation may not be observable without the activation's in some way being modified by recurrent, cooperative interactions among the ensemble of detectors activated by the stimulus. This conclusion is consistent with Braitenberg's (1978) observation that less than $1 \%$ of the input to any cortical region originates in the thalamus (i.e., the activation of cortical cells is only partially determined by their direct response to the stimulus). It also is consistent with Rose and Siebler's (1995) conclusion that large ensembles of activated detectors are not required to observe cooperativity effects. That is, they show that interconnectivity among small ensembles of neurons can result in the enhancement of their collective, neural activity. Thus, interconnectivity among the small ensemble of local, overlapping receptive fields directly activated by the same moving element could provide the bistable quality we have proposed as the basis for the state-dependent thresholds observed for the perception of single-element apparent-motion and the competing perception of nonmotion.

\section{REFERENCES}

Anstis, S. M., Giaschi, D., \& Cogan, A. I. (1985). Adaptation to apparent motion. Vision Research, 25, 1051-1062.

ANSTIS, S. M., \& MATHER, G. (1985). Effects of luminance and contrast on direction of ambiguous apparent motion. Perception, 14, 167-179.

ATtNeave, F. (1971). Multistability in perception. Scientific American, $225,62-71$

BLACKWELL, H. R. (1953). Psychophysical thresholds: Experimental studies of methods of measurement (Bulletin of the Engineering Research Institute No. 36). Ann Arbor: University of Michigan.

BRAITENBERG, V. (1978). Cortical architechtonics: General and areal. In M. A. B. Brazier \& H. Petsche (Eds.), Architechtonics of the cerebral cortex (IBRO Monograph Series, Vol. 3, pp. 443-466). New York: Raven Press.

Chang, J. J., \& Julesz, B. (1983). Displacement limits, directional anisotropy and direction versus form discrimination in random-dot cinematograms. Vision Research, 23, 639-646.

CoRso, J. F. (1967). The experimental psychology of sensory behavior. New York: Holt, Rinehart \& Winston.

EASTMAN, K. (1996). Hysteresis effects in motion- and stationaritybased figure/ground segregation [Abstract]. Investigative Ophthalmology \& Visual Science, $37, \mathrm{~S} 393$.

EASTMAN, K., \& Hock, H. S. (1995). Motion-based and stationaritybased figure/ground segregation: Effects of temporal asymmetry [Abstract]. Investigative Ophthalmology \& Visual Science, 36, S362.

Finlay, D., \& Von Grünau, M. (1987). Some experiments on the breakdown effect in apparent motion. Perception \& Psychophysics, 42, 526-534.

GILBERT, C. D. (1985). Horizontal integration in the neocortex. Trends in Neurosciences, 8, 160-165.

GREen, D. M., \& SwETS, J. A. (1966). Signal detection theory and psychophysics. New York: Wiley.

Hock, H. S., \& BALZ, G. W. (1994). Spatial scale dependent in-phase and anti-phase directional biases in the perception of self-organized motion patterns. Vision Research, 34, 1843-1861.

Hock, H. S., Balz, G. W., \& Eastman, K. (1996). Cooperative interactions and the perception of motion and stationarity for directionally ambiguous apparent motion stimuli. Perception, 25, 887-900.

Hock, H. S., Kelso, J. A. S., \& SchöneR, G. (1993). Bistability and hysteresis in the organization of apparent motion patterns. Journal of 
Experimental Psychology: Human Perception \& Performance, 19. 63-80.

Hock, H. S., Kogan, K., \& Lodes, N. (1996, September). The effect of temporal phase on the perception of apparent motion [Abstract]. Perception, 25 (Suppl.), 33.

Hock, H. S., ScHöNER, G., \& Voss, A. (1997). The influence of adaptation and stochastic fluctuations on spontaneous perceptual changes for bistable stimuli. Perception \& Psychophysics, 59, 509-522.

Johansson, G. (1950). Configurations in event perception. Uppsala: Almqvist \& Wiksell.

Kolers, P. A. (1972). Aspects of motion perception. Oxford, U.K.: Pergamon.

NaWrot, M., \& SekUler, R. (1990). Assimilation and contrast in motion perception: Explorations in cooperativity. Vision Research, 30 , 1439-1451.

PetersiK, J. T. (1990). Global cooperativity of the short-range process in apparent movement: Evidence obtained with contour-containing stimuli. Perception \& Psychophysics, 47, 360-368.

RAYMOND, J., \& BRADDICK, O. (1996). Responses to opposed directions of motion: Continuum or independent mechanisms. Vision Research, 36, $1931-1937$.

Rose, G., \& SiEbler, M. (1995). Cooperative effects of neuronal ensembles. Experimental Brain Research, 106, 106-110.

SCHÖNER, G., \& Hock, H. S. (1995). Concepts for a dynamical theory of perceptual organization: An example from apparent movement. In P. Kruse \& M. Stadler (Eds.), Ambiguity in mind and nature (pp. 275310). Berlin: Springer Verlag.

SEKULER, R. (1996). Motion perception: A modern view of Wertheimer's 1912 monograph. Perception, 25, 1243-1258.

WERTHEIMER, M. (1912). Experimentelle Studien über das Sehen von Bewegung [Experimental studies of the perception of movement].
Zeitschrift für Psychologie und der Physiologie der Sinnesorgane, 61, 161-265.

Williams, D., Phillips, G., \& Sekuler, R. (1986). Hysteresis in the perception of motion direction as evidence for neural cooperativity. Nature, 324, 253-255.

WiLLIAMS, D., \& SeKULER, R. (1984). Coherent global motion percepts from stochastic local motions. Vision Research, 24, 55-62.

\section{NOTES}

1. Hock, Schöner, and Voss (1997) have shown that adaptation can influence the rate of switching between bistable percepts without being the direct cause of the switches.

2 . The single-element apparent-motion paradigm can be further generalized by varying the relative temporal phase of luminance change for the two elements (Hock, Kogan, \& Lodes, 1996; Johansson, 1950). The relative phase is $180^{\circ}$ in standard apparent motion.

3. The values of $L_{\mathrm{m}}$ are indicated as approximate because having to choose among 256 gray levels prevented the continuous variation in luminance values. This made it impossible to make $L_{\mathrm{m}}$ exactly the same for each $L_{1} / L_{2}$ pair.

4. Hysteresis involving motion/nonmotion transitions has recently been reported for figure/ground segregation based on the perception of a moving versus a stationary figure (Eastman, 1996).

5. Another possibility is that the cooperativity effects arise from interactions with receptive fields that are more sensitive to position than to motion (Eastman \& Hock, 1995).

(Manuscript received July 1, 1996; revision accepted for publication October 16, 1996.) 